\title{
Immunization with virus-like particles conjugated to CIDRa1 domain of Plasmodium falciparum erythrocyte membrane protein 1 induces inhibitory antibodies
}

\author{
Charlotte Harmsen ${ }^{1,2}$, Louise Turner ${ }^{1,2}$, Susan Thrane ${ }^{1,2}$, Adam F. Sander ${ }^{1,2}$, Thor G. Theander ${ }^{1,2}$ \\ and Thomas Lavstsen ${ }^{1,2^{*}}$ (D)
}

\begin{abstract}
Background: During the erythrocytic cycle, Plasmodium falciparum malaria parasites express $P$. falciparum Erythrocyte Membrane Protein 1 (PfEMP1) that anchor the infected erythrocytes (IE) to the vascular lining of the host. The CIDRa1 domain of PfEMP1 is responsible for binding host endothelial protein C receptor (EPCR), and increasing evidence support that this interaction triggers severe malaria, accounting for the majority of malaria-related deaths. In high transmission regions, children develop immunity to severe malaria after the first few infections. This immunity is believed to be mediated by antibodies targeting and inhibiting PfEMP1, causing infected erythrocytes to circulate and be cleared in the spleen. The development of immunity to malaria coincides with acquisition of broad antibody reactivity across the CIDRa1 protein family. Altogether, this identifies CIDRa1 as an important vaccine target. However, the antigenic diversity of the CIDRa1 domain family is a challenge for vaccine development.
\end{abstract}

Methods: Immune responses in mice vaccinated with Virus-Like Particles (VLP) presenting CIDRa1 antigens were investigated. Antibody reactivity was tested to a panel of recombinant CIDRa1 domains, and the antibodies ability to inhibit EPCR binding by the recombinant CIDRa1 domains was tested in Luminex-based multiplex assays.

Results: VLP-presented CIDRa1.4 antigens induced a rapid and strong IgG response capable of inhibiting EPCR-binding of multiple CIDRa1 domains mainly within the group A CIDRa1.4-7 subgroups.

Conclusions: The study observations mirror those from previous CIDRa1 vaccine studies using other vaccine constructs and platforms. This suggests that broad CIDRa1 antibody reactivity may be achieved through vaccination with a limited number of CIDRa 1 variants. In addition, this study suggest that this may be achieved through vaccination with a human compatible VLP vaccine platform.

Keywords: Malaria, Plasmodium falciparum, Vaccine, Virus-like particle, Antigenic diversity, PfEMP1, CIDRa1

\footnotetext{
*Correspondence: thomasl@sund.ku.dk

${ }^{1}$ Centre for Medical Parasitology, Department of Immunology and Microbiology (ISIM), Faculty of Health and Medical Sciences, University of Copenhagen, Copenhagen, Denmark

Full list of author information is available at the end of the article
}

\section{Background}

Malaria caused by Plasmodium falciparum is still a leading disease accounting for considerable under-five mortality in sub-Saharan Africa. In areas of moderate to high transmission intensity, severe life-threatening malaria mainly affects infants and toddlers, as immunity against severe disease is acquired at a young age

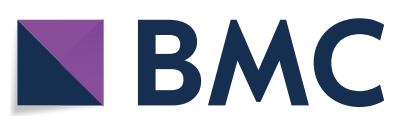

(c) The Author(s) 2020. This article is licensed under a Creative Commons Attribution 4.0 International License, which permits use, sharing, adaptation, distribution and reproduction in any medium or format, as long as you give appropriate credit to the original author(s) and the source, provide a link to the Creative Commons licence, and indicate if changes were made. The images or other third party material in this article are included in the article's Creative Commons licence, unless indicated otherwise in a credit line to the material. If material is not included in the article's Creative Commons licence and your intended use is not permitted by statutory regulation or exceeds the permitted use, you will need to obtain permission directly from the copyright holder. To view a copy of this licence, visit http://creativeco mmons.org/licenses/by/4.0/. The Creative Commons Public Domain Dedication waiver (http://creativecommons.org/publicdomain/ zero/1.0/) applies to the data made available in this article, unless otherwise stated in a credit line to the data. 
after a few malaria episodes [1-3]. Young children who have acquired immunity to severe infections are still susceptible to less severe malaria episodes and immunity against these gradually forms during childhood [4]. This development of immunity can be explained by a gradual acquisition of IgG against variable polymorphic proteins expressed on the surface of infected erythrocytes [5-9]. Members of the $P$. falciparum Erythrocyte Membrane Protein 1 (PfEMP1) family are considered main targets of this immunity. These proteins are anchored in the erythrocyte membrane exposing their large $\mathrm{N}$-terminal to engage with receptors on endothelial cells (reviewed in [10]). This permits an effective sequestration of infected erythrocytes to the endothelial lining and allows these cells to escape blood flow and splenic filtration. On the erythrocyte surface, PfEMP1 is accessible to antibodies functionally inhibiting the binding between the infected erythrocyte and the endothelium. These antibodies are thought to be important mediators of immunity [11-14].

Each parasite genome contains 50-60 PfEMP1encoding var genes, which differ in sequence within and between parasites, and of which each parasite will express only one during the erythrocytic cycle [15]. Despite their extensive sequence diversity, the distribution of different PfEMP1 types is similar in all parasites [16]. PfEMP1s are composed of two to nine Duffy Binding-like (DBL) and cysteine-rich interdomain region (CIDR) domains, and in most PfEMP1 the second domain from the $\mathrm{N}$-terminus is a CIDR domain [16]. These domains have diversified to confer binding to either endothelial protein $C$ receptor (EPCR) (CIDR $\alpha 1$ domains) [17], CD36 (CIDR $\alpha 2-6$ domains), or unknown receptors (CIDR $\beta / \gamma / \delta$ domains). These mutually exclusive binding phenotypes are maintained by chromosomal separation of the encoding genes, with so-called group B and C var genes encoding CD36-binding PfEMP1 and group A genes encoding EPCR-binding PfEMP1 and CIDR $\beta / \gamma / \delta$-domain PfEMP1. In addition, most parasites carry one to three variants of the so-called DC8 group B/A chimeric var genes also encoding EPCR-binding PfEMP1 [18].

Numerous studies have linked EPCR-binding parasites, or parasites expressing CIDR $\alpha 1$-PfEMP1, with development of severe malaria in including when it is presenting as cerebral malaria and severe anemia. No other PfEMP1 domain is consistently associated with severe malaria pathology [19-21]. However, CIDR $\alpha 1-P f E M P 1$ are large multi-domain molecules, and it is likely that endothelial receptor-interactions of some accompanying domains act in concert with EPCR-binding to promote parasite survival. For example, current evidence suggests that supplementary binding to ICAM1 or HABP1 is associated with EPCR binding by group A [21, 22] and B/A PfEMP1 [23], respectively. PfEMP1 binding to EPCR abrogates
EPCR-mediated conversion of protein $\mathrm{C}$ to activated protein $\mathrm{C}$, which promotes a pro-inflammatory state of the endothelium prone to thrombin-induced expression of endothelial cell adhesion molecules including the Intercellular Adhesion Molecule 1 (ICAM1) [24, 25].

All CIDR $\alpha 1$ domains adopt a similar fold in order to bind EPCR with high affinity [26]. The EPCR binding mechanism of the CIDR $\alpha 1$ domain mimics that of activated protein $C$ to EPCR, indicating that an evolutionary stalemate has been reached, in which neither the parasite or human protein can mutate its basic structure without compromising its vital function. However, immune selection pressure has imposed extensive sequence diversity across surface exposed amino acids on the CIDR $\alpha 1$ domain, albeit limited to variation maintaining structure and the physio-chemical properties of the amino acids directly interacting with EPCR. As a result, EPCR-binding CIDR $\alpha 1$ sequence variants distribute into six subgroups, CIDR $\alpha 1.1$ and CIDR $\alpha 1.4-1.8$, of which CIDR $\alpha 1.1$ and 1.8 are found in group B/A PfEMP1 and the rest among group A PfEMP1. The EPCR-binding site is essentially comprised of a $\sim 25$ amino acid kinked alpha-helix structure protruding from a core triple helix structure [26]. Across the $19 \mathrm{kDa}$ minimal binding domain of CIDR $\alpha 1$, sequences are pairwise $40-100 \%$ identical. The average sequence identity within CIDR $\alpha 1$ subgroups (e.g. 1.1) is 69-72\%, but short amino acid stretches of high similarity are shared across members of different subtypes [26].

Experimental immunization of mice and rats with single or multiple soluble CIDR $\alpha 1$ proteins, or adenovirus inducing in vivo secretion of CIDR $\alpha 1$ protein, readily elicit EPCR binding-inhibitory IgG to cognate antigens, as well as to CIDR $\alpha 1$ variants of the same CIDR $\alpha 1$ subtype as the used immunogen [27, 28]. The most heterogeneous of CIDR $\alpha 1$ subtypes is the CIDR $\alpha 1.4$ and CIDR $\alpha 1.7$ domains, to which it appears to be particularly difficult to elicit cross-ractive antibodies. Vaccines presenting antigens on Virus-Like Particles (VLP) have been shown to induce high tittered, long lasting functional IgG responses in humans $[29,30]$ and can drive immune responses even to weakly immunogenic or self-antgens $[31,32]$. We, and others, have recently developed a technique that allows effective coupling of complex proteins to pre-formed VLP and demonstrated that the IgG response to the proteins coupled is potent, functional, and durable even with self-antigens [31, 33, 34]. Here, VLP-CIDR $\alpha 1.4$ vaccines adjuvanted with aluminum hydroxide elicited stronger immune responses than a similar soluble CIDR $\alpha 1$ vaccine in Freund's incomplete adjuvant, and antibodies reactive and functionally inhibitory across group A CIDR $\alpha 1$ variants was elicited from immunization with a single CIDR $\alpha 1$ antigen variant. 


\section{Methods}

\section{Recombinant protein and VLP production}

Proteins were produced in baculovirus-infected High Five cells as previously described [35]. CIDR $\alpha 1$ proteins derived from the HB3var03 PfEMP1 sequence were genetically fused with the SpyCatcher [36] protein sequence at the $\mathrm{N}$-terminal or $\mathrm{C}$-terminal and a Strep-tag II sequence in the opposite terminal (total size $32 \mathrm{kDa}$ ): catCIDR $\alpha 1.4$ (SpyCatcher-CIDR $\alpha 1.4-$ Strep-tag II), and CIDR $\alpha 1.4$ cat (Strep-tag II-CIDR $\alpha 1.4-S p y C a t c h e r)$.

SpyT-VLPs formed by 180 capsid units of the Acinetobacter bacteriophage AP205 and displaying one SpyTag per capsid unit, were produced as previously described [31]. In brief, the SpyTag sequence (AHIVMVDAYKPTK) was fused to the N-terminus of each capsid using a flexible linker (GSGTAGGGSGS). The construct was expressed in E. coli One Shot BL21 StarTM (DE3) cells (Thermo Scientific) and purified by ultracentrifugation using an OptiprepTM density gradient (Sigma). Naked VLP formed from AP205 capsids with no SpyTag were produced using the same method.

Assembled SpyTagged VLP and soluble SpyCatcherfused CIDR $\alpha 1.4$ antigen were mixed at a 1:1 molar ratio (VLP capsid per antigen) and incubated over night at $4{ }^{\circ} \mathrm{C}$, forming a $\sim 48 \mathrm{kDa}$ band on SDS-PAGE under reducing conditions (Fig. 1). Uncoupled antigen was not removed from the vaccine before administration.

Dynamic light scattering (DLS) was performed as previously described [31]. Briefly, the distribution of VLP particle sizes was acquired at $658 \mathrm{~nm}, 25{ }^{\circ} \mathrm{C}$ (WYATT Technology, DynaPro NanoStar). Each VLP preparation was measured twice with 20 runs and the estimated diameter of the main particle population and the percent polydispersity (\%Pd) was calculated.

\section{Mice and immunizations}

Female BALB/c mice (Taconic, Denmark), 7-8 weeks old, were immunized by intramuscular injection three times, at 3-week intervals. The mice were given $5 \mu \mathrm{g}$ CIDR $\alpha 1.4$ antigen per mouse per immunization, administered as either bound to $2.5 \mu \mathrm{g}$ SpyTag-VLP or without any VLP. In total 16 mice were distributed between the four groups: VLP-catCIDR $\alpha 1.4$ (five mice), CIDR $\alpha 1.4$ cat-VLP (five mice), catCIDR $\alpha 1.4$ (three mice), and CIDR $\alpha 1.4$ cat (three mice). VLP-coupled antigen was administered with Alhydrogel (2\%) (Brenntag) added to the vaccine formulation $1 \mathrm{~h}$ prior to immunization, whereas antigens with no VLP was administered with Freund's incomplete adjuvant (1:1, W/W). Serum was collected 2 weeks after each immunization. Total IgG was purified using GammaBind Plus Sepharose (BD Biosciences) according to the manufacturer's protocol.

\section{ELISA analyses}

Serum levels of antigen specific IgG were measured as previously described [31]. In brief, plates were coated with $5 \mu \mathrm{g} / \mathrm{mL} 30 \mathrm{kDa}$ HIS-tagged HB3var03 CIDR $\alpha 1.4$ protein [28]. The wells were blocked with $1 \%$ BSA buffer.
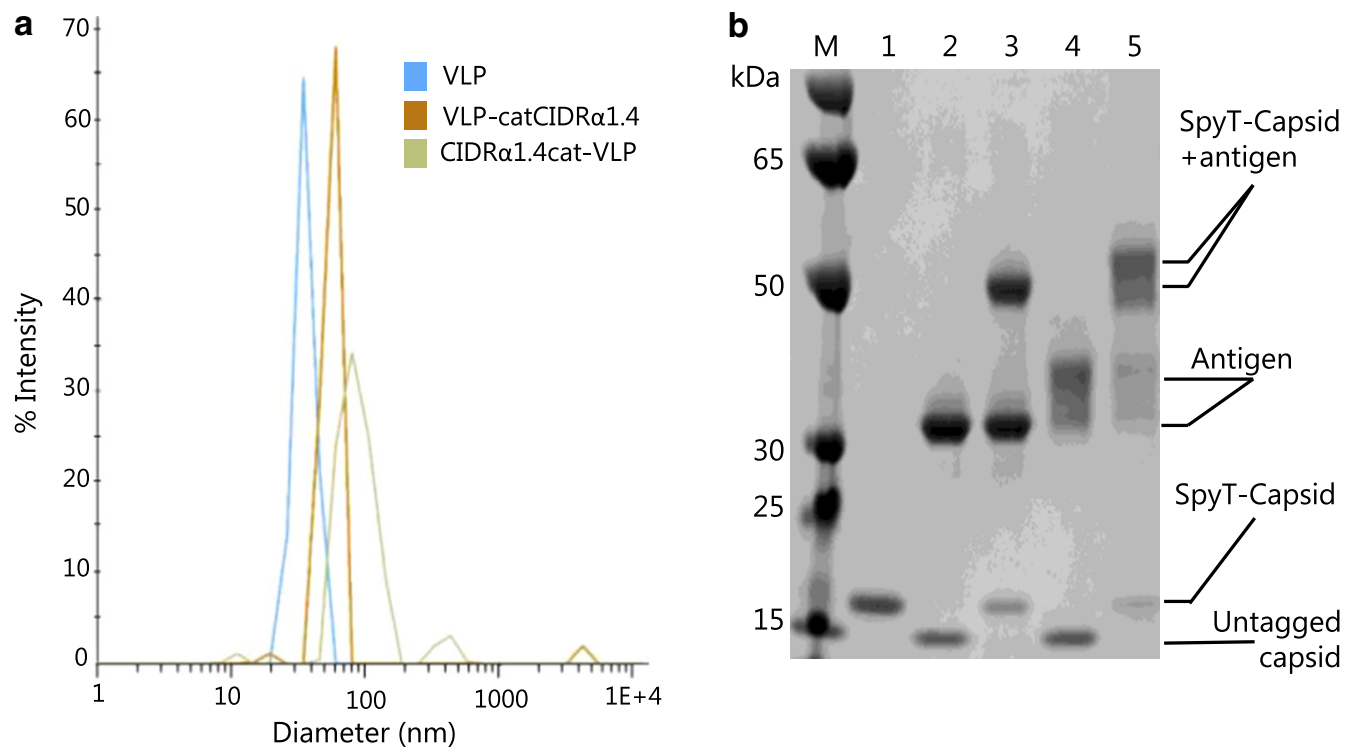

Fig. 1 Characterization of VLP-antigen conjugated vaccines. a Dynamic light scattering (DLS) showing SpyT-VLP (blue) with an average size of 37 $\mathrm{nm}$ (12.18\% polydispersity, Pd). After coupling VLP to catCIDRa1.4 (brown) or CIDRa1.4cat (green) the size increases to $56 \mathrm{~nm}$ (12.19\%Pd) and $88 \mathrm{~nm}$ (27.83\%Pd), respectively. b SDS-gel loaded with catCIDRa1.4 or CIDRa1.4cat antigens (32kDa) mixed with SpyT-VLP (16kDa) or naked VLP (8kDa): 1. SpyT-VLP; 2. Naked VLP + catCIDRa1.4; 3. SpyT-VLP + catCIDRa1.4; 4. Naked VLP + CIDRa1.4cat; 5. SpyT-VLP + CIDRa1.4cat 
Mouse serum was added in a series of three-fold dilutions starting from 1:50. Secondary antibody (HRP-conjugated anti mouse IgG (Life Technologies, Denmark)) was diluted 1:3000 and color reactions were developed for $7 \mathrm{~min}$ by adding o-phenylenediamine substrate.

\section{IgG reactivity and EPCR-binding inhibition of diverse CIDRa1 domains}

IgG reactivity to a panel of 43 recombinant CIDR $\alpha 1$ domains coupled to Luminex microspheres was measured as in [37]. In short, serum was diluted 1:80, and IgG reactivity was detected using secondary phycoerythrin (PE)-conjugated antibody diluted to 1:3000. For assessing EPCR-binding inhibition, purified IgG from each mouse within a vaccine group was pooled and incubated at $0.25 \mathrm{mg} / \mathrm{mL}$ IgG with the CIDR $\alpha 1$-conjugated Luminex microspheres for $30 \mathrm{~min}$ at room temperature. After washing with standard Luminex buffers, microspheres were incubated with $4 \mu \mathrm{g} / \mathrm{mL}$ biotinylated recombinant EPCR for $30 \mathrm{~min}$ at room temperature. EPCR-binding was detected using PE-conjugated streptavidin. The CIDR $\alpha 1$ protein variants with low binding to EPCR when coupled to microspheres were excluded from the inhibition study leaving 34 variants tested.

\section{Data analysis}

Data were analysed using Microsoft Excel and STATA (StataCorp). Non-parametric statistical tests was used as indicated in the result section. IgG titers were calculated as area under curve using STATA14 using the pkexamine command using dilution as time parameter over 11 dilution steps, ELISA OD as concentration parameter and the trapezoidal rule.

\section{Protein sequences \\ catCIDRa 1.4 (SpyC-HB3var03-Strep-Tag II) (linkers in lower case)}

GAMVDTLSGLSSEQGQSGDMTIEEDSATHIKFSKRD EDGKELAGATMELRDSSGKTIST WISDGQVKDFY LYPGKYTFVETAAPDGYEVATAITFTVNEQGQVT VNGKATKGDAHIggsKITSFDEFFDFWVRKLLID TIKWETELTYCINNTDVTDCNKCNKNCVCFD KWVKQKEDEW TNIMKLFT NKHDIPKKYYLN INDLFDSFFFQVIYKFNEGEAKWNELKENLKKQIASS KANNGTKDSEAAIKVLFNHIKEIATICKDNNTNEGrtg WSHPQFEK.

\section{CIDRa1.4cat (Strep-tag-II-HB3var03-SpyC) (linkers in lower case)}

WSHPQFEKrtgKITSFDEFFDFWVRKLLIDTIKW ETELTYCINNTDVTDCNKCNKNCVCFDKWVKQKE DEWTNIMKLFTNKHDIPKKYYLNINDLFDSFFFQVIY KFNEGEAKWNELKENLKKQIASSKANNGTKD-
SEAAIKVLFNHIKEIATICKDNNTNEGggsGAMV DTLSGLSSEQGQSGDMTIEEDSATHIKFSKRDEDGK ELAGATMELRDSSGKTISTWISDGQVKDFYLYPGK YTFVETAAPDGYEVATAITFTVNEQGQVTVNGKAT KGDAHI.

\section{Results}

\section{Production VLP-based CIDRa1 vaccines}

Two recombinant CIDR $\alpha 1$ proteins both derived from the HB3var03 PfEMP1 CIDR $\alpha 1.4$ sequence were produced with a SpyCatcher protein sequence in either the $\mathrm{N}$-terminal or the C-terminal (catCIDR $\alpha 1.4$ and CIDR $\alpha 1.4$ cat, respectively) (Fig. 1). The recombinant proteins were coupled to bacteriophage AP205 SpyTagVLP particles. These particles form spontaneously from 180 AP205 capsid proteins, each with a genetically fused SpyTag in the N-terminus. By mixing a recombinant SpyCatcher-fused CIDR $\alpha 1.4$ protein with the pre-formed VLP, the SpyTags and SpyCatchers formed covalent bonds, resulting in VLP coupled with CIDR $\alpha 1.4$ antigen (Fig. 1).

\section{Analysis of the IgG reactivity to the CIDRa1 domain used as immunogen}

The two VLP based CIDR $\alpha 1.4$ vaccines and two vaccines containing the corresponding soluble proteins were administered by intramuscular injection to BALB/C mice over three immunizations with 3-week intervals. Two groups of five mice received catCIDR $\alpha 1.4$ or CIDR $\alpha 1.4$ cat displayed on VLP in aluminum hydroxide and two groups of three mice received catCIDR $\alpha 1.4$ or CIDR $\alpha 1.4$ cat without VLP but with Freund's incomplete adjuvant.

Serum collected 2 weeks after each of the three immunizations was assessed for induction of IgG binding to cognate HB3var03 CIDR $\alpha 1.4$ domains (without the SpyCatcher tag of the immunogens) in ELISA. The CIDR $\alpha 1.4-V L P$ vaccines in aluminum hydroxide induced a faster and stronger IgG response against the cognate HB3var03 CIDR $\alpha 1.4$ antigen than the vaccines with soluble antigen in Freund's incomplete adjuvant (Fig. 2). The effect was most pronounced after the first immunization (Fig. 2, 1st bleed).

\section{Analysis of IgG reactivity to sequence diverse CIDRa1 domain variants}

Next, the reactivity of serum IgG to different CIDR $\alpha 1$ protein variants $(\mathrm{N}=43)$, and the ability to inhibit binding between 34 of these variants and EPCR with total purified IgG, was tested using a Luminex based multiplex-system. Luminex microspheres coupled with individual CIDR $\alpha 1$ domains were mixed and used in assays measuring the amount of IgG binding each antigen (IgG 

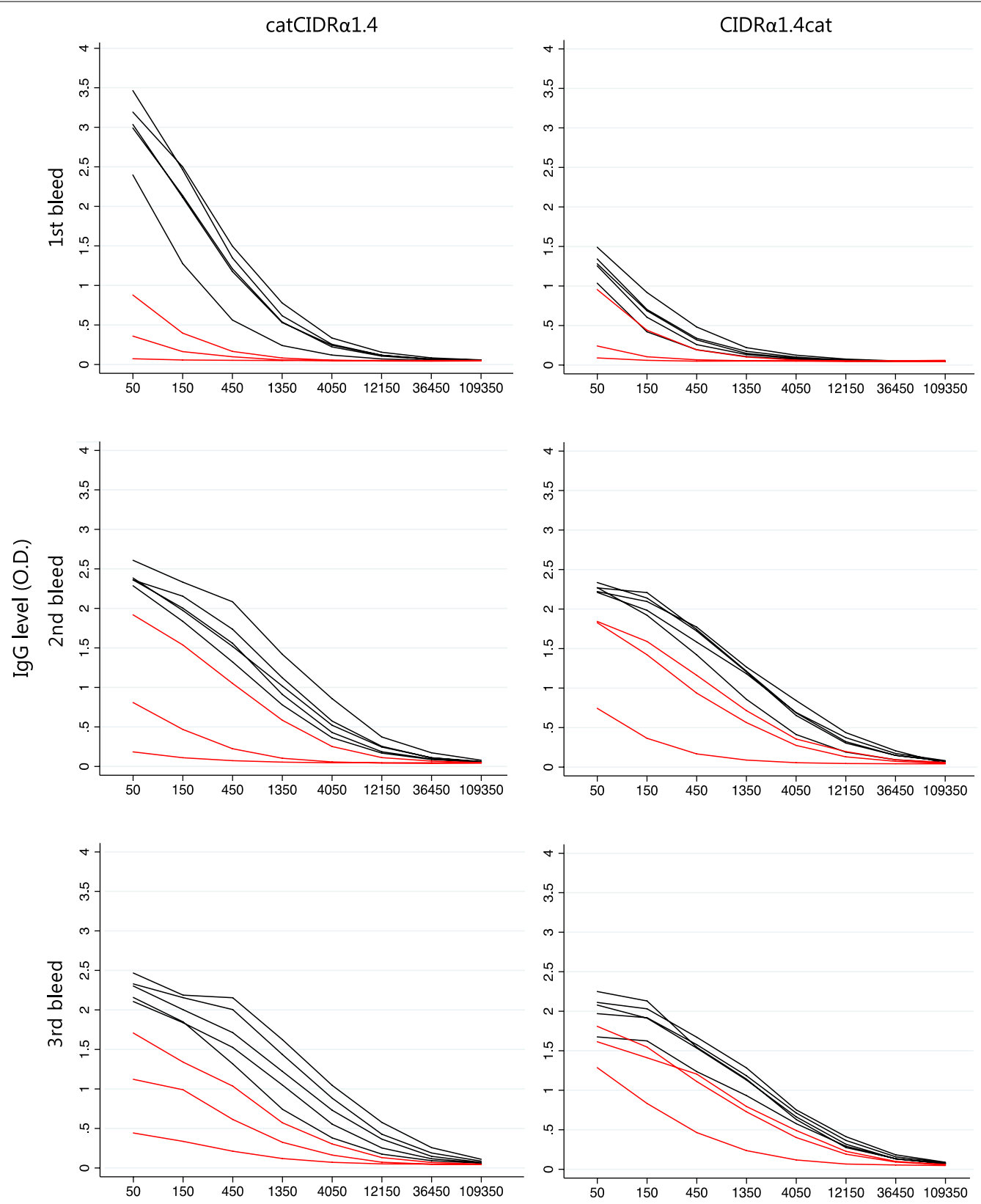

Dilution

\begin{tabular}{|c|c|c|c|c|c|c|c|}
\hline \multicolumn{2}{|c|}{ Area under the curve } & \multicolumn{2}{|c|}{ catCIDRa1 } & \multicolumn{2}{|c|}{ CIDRa1cat } & \multicolumn{2}{|c|}{$\mathrm{P}$ (On vs No VLP) } \\
\hline & & mean & $\pm S D$ & mean & $\pm S D$ & catCIDRa1 & CIDRa1cat \\
\hline \multirow{2}{*}{ 1st bleed } & On VLP & 7.5 & 1.5 & 2.9 & 0.5 & \multirow{2}{*}{0.03} & \multirow{2}{*}{0.07} \\
\hline & No VLP & 2.3 & 1.4 & 1.9 & 0.8 & & \\
\hline \multirow{2}{*}{ 2nd bleed } & On VLP & 8.3 & 1.1 & 8.5 & 0.7 & \multirow{2}{*}{0.003} & \multirow{2}{*}{0.009} \\
\hline & No VLP & 2.9 & 2.5 & 4.5 & 2.4 & & \\
\hline \multirow{2}{*}{ 3rd bleed } & On VLP & 8.7 & 1.5 & 8.0 & 0.8 & \multirow{2}{*}{0.002} & \multirow{2}{*}{0.01} \\
\hline & No VLP & 3.5 & 1.9 & 5.2 & 1.6 & & \\
\hline
\end{tabular}


(See figure on previous page.)

Fig. 2 Anti-CIDRa1.4 IgG. ELISA titers elicited in mice vaccinated with catCIDRa1.4 or CIDRa1.4cat. Each line represents the titration of serum from a mouse. Black lines indicate serum from mice immunized with CIDRa1.4-VLP in aluminum hydroxide. Red lines indicate mice immunized with soluble CIDRa1.4 in Freund's incomplete adjuvant. Table shows titers calculated as area under the curve for each vaccine group (mean \pm standard deviation), P values from t-tests

level, Mean Fluorescence Intensity (MFI)) or the ability of EPCR to bind CIDR $\alpha 1$ on the microsphere in the presence of IgG (inhibition of EPCR-binding, Inhibition (\%)). Figure 3 shows the antibody levels measured in serum from individual mice, and the inhibitory activity of purified total IgG pooled from animals receiving the same vaccine (both with serum collected 2 weeks after the third immunization). This analysis confirmed that the VLP vaccines induced higher levels of IgG to the cognate immunogen (HB3var03) than the vaccines with soluble antigen in Freund's incomplete adjuvant (Fig. 3a,
$\mathrm{P}=0.03$ and $\mathrm{P}=0.05$ for catCIDR $\alpha 1.4$ and CIDR $\alpha 1.4$ cat, respectively; Wilcoxon rank-sum test). Furthermore, all vaccines induced IgG with high capacity to inhibit EPCRbinding of the cognate CIDR $\alpha 1$ immunogen (Fig. 3b).

When assessing the serum reactivity and EPCR-binding inhibitory potential of purified IgG to the panel of CIDR $\alpha 1$ domains not included in the vaccine (Fig. 3c, d), reactivity to and inhibition of specific variants varied considerably. However, there was no overall difference in these parameters between the VLP coupled and the soluble vaccines. When plotting the inhibition data for each

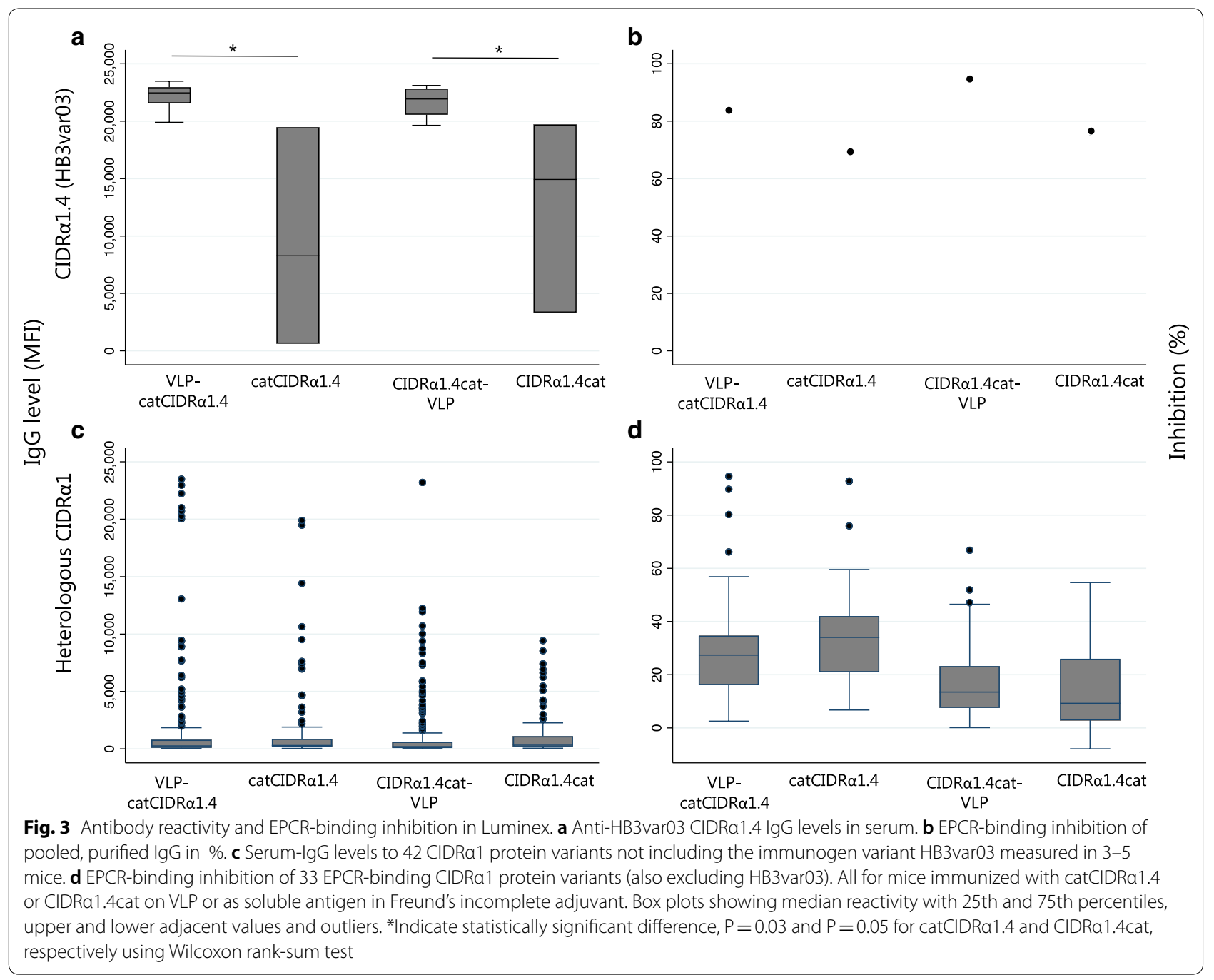



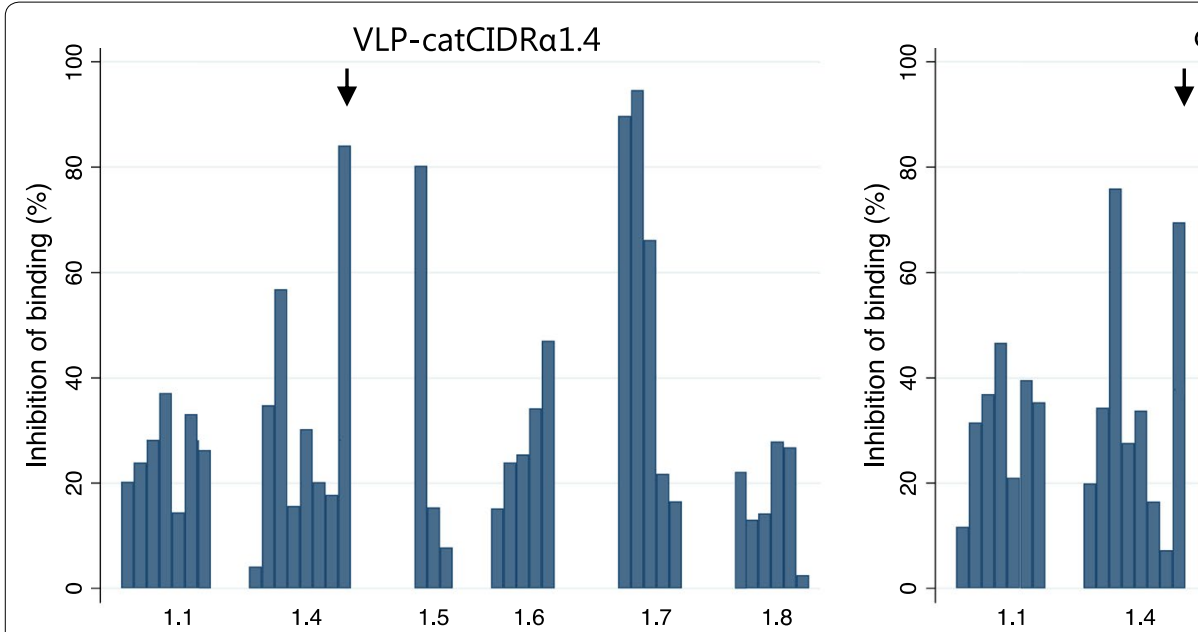

catCIDRa1.4

$\downarrow$
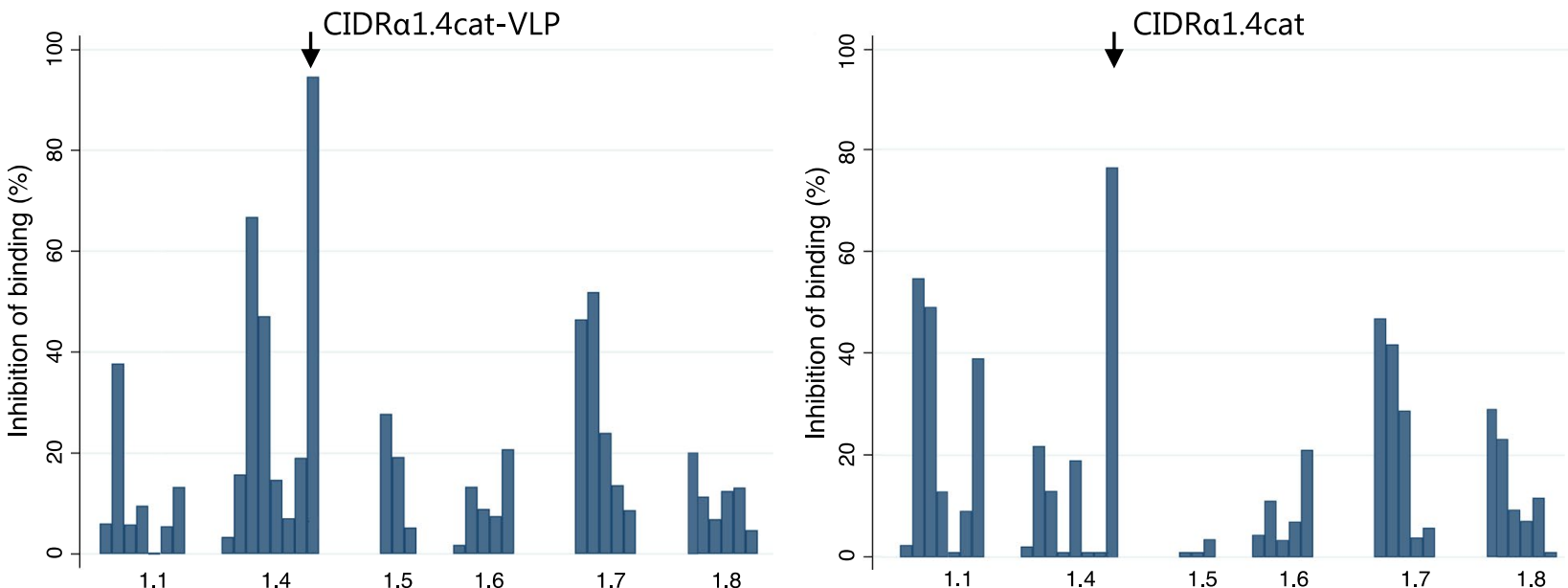

Fig. 4 Inhibition of EPCR-binding of specific CIDRa1 domain variants. EPCR-binding inhibition (\%) of 34 recombinant CIDRa1 domains by purified lgG pooled from mice immunized with VLP-catCIDRa1.4, catCIDRa1.4, CIDRa1.4cat-VLP or CIDRa1.4cat. Each bar represents one specific recombinant CIDRa1 domain variant annotated according to its domain subtype. Arrows indicate the HB3var03 variant used as immunogen. Order of CIDRa1 protein variants tested is shown as listed in Fig. 6 a

specific CIDR $\alpha 1$ domain variant assayed (Fig. 4), inhibition was consistently observed for some domains. Overall, the CIDR $\alpha 1$ variants to which inhibitory antibodies were elicited were common to the four vaccines, albeit inhibition was somewhat more pronounced for IgG elicited by the catCIDR $\alpha 1.4$ antigens. If $40 \%$ inhibition was applied as a cutoff the VLP-catCIDR $\alpha 1.4$ vaccine induced IgG, inhibitingsix of 20 tested group A CIDR $\alpha 1$ variants (CIDR $\alpha 1.4-7)$ and none of 13 CIDR $\alpha 1.1 / 1.8$ variants tested from the group B/A PfEMP1 (a.k.a. DC8 PfEMP1). IgG elicited by the soluble catCIDR $\alpha 1.4$ vaccine inhibited seven of 20 heterologous CIDR $\alpha 1$ variants from group $\mathrm{A}$, and two of 13 CIDR $\alpha 1.1 / 1.8$ variants. Interestingly, in particular the binding of CIDR $\alpha 1.7$ domains, which are closest related to CIDR $\alpha 1.4$ used as immunogen, were inhibited. Raw data given in Additional File 1.
There was a clear but not absolute correlation between IgG level to and the IgG binding inhibition of the single CIDR $\alpha 1$ domains (Ks $=0.67, \mathrm{P}=0.0001$, Spearman's rank-order correlation) (Fig. 5). For many domains, however, there was a considerable antibody recognition (MFI $>1000)$ without high inhibitory capacity. A particular high reactivity without strong inhibition was seen for two specific CIDR $\alpha 1.4$ variants (CIDR $\alpha 1.4$ 1983-5 and CIDRo1.4_DD2var32). This may indicate that elicited antibodies targeted shared non-inhibitory epitopes of the domains or that the affinity of the elicited antibodies was insufficient to compete with EPCR.

Figure 6 shows the sequence relation between the studied CIDR $\alpha 1$ variants. Overall sequence similarity between immunogen and antigen was not clearly associated with antigen inhibition, albeit that the most similar domain, sharing $88 \%$ amino acids with the immunogen 


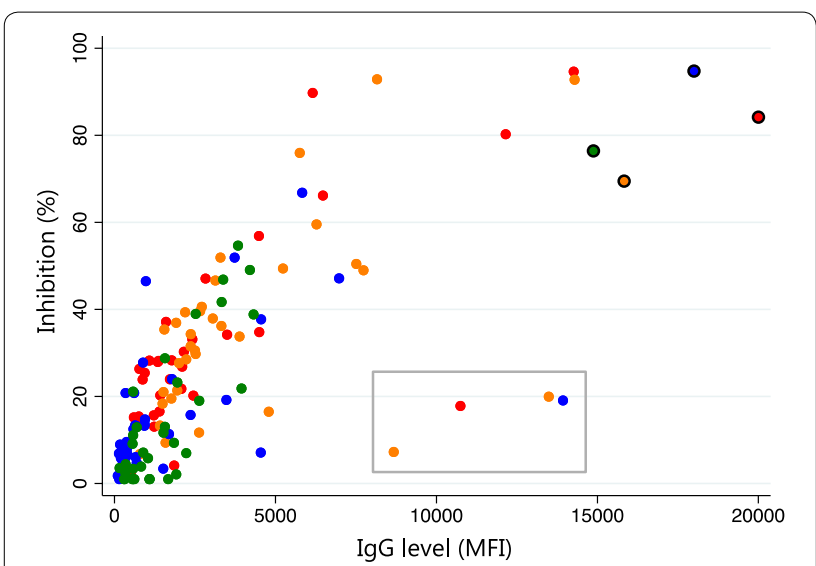

Fig. 5 Correlation between anti-CIDRa1 lgG levels and EPCR-binding inhibitory capacity of CIDRa1 domains in pooled lgG purified from mice immunized with VLP-catCIDRa1.4. Each dot represents one EPCR-binding CIDRa1 domain and colors represent each of the four immunization schemes (34 domains $\times 4$ immunization groups): VLP-catCIDRa1.4 (red), catCIDRa1.4 (orange), CIDRa1.4cat-VLP (blue), and CIDRa1.4cat (green). The immunogen CIDRa1.4 variant is outlined. The box includes indicates data from three CIDRa1.4_ DD2var32 and one CIDRa1.4_1983-5 domain

variant, was the strongest inhibited. No distinct amino acid sequence trait shared between inhibited CIDR $\alpha 1$ variants could be identified from sequence alignments (from visual inspection and with SigniSite, http://www. cbs.dtu.dk/services/SigniSite/). However, overall it was mainly CIDR $\alpha 1$ variants within group $A$, and in particular the CIDR $\alpha 1.7$ variants, that were inhibited.

\section{Discussion}

Increasing evidence support that the interaction between the CIDR $\alpha 1$ domains of PfEMP1 and EPCR is key to the development of severe $P$. falciparum malaria [38]. Parasites causing severe malaria mainly express PfEMP1 binding EPCR and increasing proportions of EPCR-binding parasites appears to be associated with increase with severity of symptoms. No other PfEMP1 domain or trait shows this association [20, 21, 39-44], despite all PfEMP1 carrying additional domains capable of interacting with host receptors. This strong association may be tied to the observation that CIDR $\alpha 1$ binding to EPCR disrupts EPCR function, triggering endothelial inflammation and vascular leakage, characteristic for the pathology of severe malaria [45]. Children in malaria endemic areas develop immunity to severe malaria after relatively few infections coinciding with the acquisition of antibodies to CIDR $\alpha 1[9,46]$. For these reasons, CIDR $\alpha 1$ is considered an important target for vaccines protecting against clinical and particularly severe malaria [47].

However, the challenge to vaccine development is the antigenic diversity of the CIDR $\alpha 1$ domains. Based on amino acid sequence, the domains can be divided into six subclasses (1.1, and 1.4-1.8), each of which contain many different sequence variants. Despite the considerable amino acid variation, the protein structure and molecular basis of the interaction with EPCR is maintained across CIDR $\alpha 1$ domains [26]. Immunity to severe malaria is acquired relatively fast, which suggests that natural infection can induce IgG, targeting a relatively broad panel of CIDR $\alpha 1$ types. This notion is supported by the fact that IgG purified from malaria-exposed children and by affinity for a single CIDR $\alpha 1.1$ variant, can inhibit the EPCRbinding of distant CIDR $\alpha 1.4$ variants and vice versa [26]. The complexity of these antibody pools remains unknown. However, as short 63-mer peptides spanning the EPCR binding site was used for affinity purification in these studies, the data suggest that inhibitory antibodies interact with similar epitopes at or near the EPCR-binding site of different CIDR $\alpha 1$ variants [26].

In the present study, CIDR $\alpha 1.4$ immunization readily produced antibodies inhibiting the EPCR-binding of the CIDR $\alpha 1.4$ variant present in the vaccines as well as other CIDR $\alpha 1$ variants typically within the same subgroup as the immunogen. This was also seen in previous studies $[27,28]$, where animals were vaccinated with soluble recombinant CIDR $\alpha 1$ domains or live attenuated adenovirus inducing in vivo CIDR $\alpha 1$ protein secretion. In these studies, induction of cross-reactive antibodies was most clearly demonstrated by vaccination using two CIDR $\alpha 1.1$ domains resulting in antibodies reactive to 11 of 14 group $\mathrm{B} / \mathrm{A}(\mathrm{CIDR} \alpha 1.1$ and CIDR $\alpha 1.8)$ antigen variants [27]. The group B/A PfEMP1 are encoded by a set of chimeric var genes originating from group A and B var gene recombination events $5^{\prime}$ to the CIDR $\alpha 1$ sequence. Due to the opposite directions of transcription of group $\mathrm{A}$ vs. B and B/A var genes, these var gene subfamilies are likely to have evolved separately. Within group A PfEMP1, CIDR $\alpha 1$ domains have diverged into groups, with CIDR $\alpha 1.4$ and 1.7 variants being the closest related. Within subgroups CIDR $\alpha 1$ domains are typically $65-75 \%$ pairwise identical [16]. Between group $A$ and B/A, CIDR $\alpha 1$ variants are on average $46 \%$ pairwise identical. The data presented here, indicate that immunization with a group A CIDR $\alpha 1$ mainly elicited cross-binding inhibitory antibodies against other group A CIDR $\alpha 1$ variants. This suggests that group B/A and group A CIDR $\alpha 1$ domains, to a certain extent comprise two separate serogroups. It was not possible from the present data to identify shared specific epitopes responsible for recognition and inhibition across variants.

Vaccines based on VLP-conjugated antigens are very effective in inducing long-lived functional antibodies. The best examples are the HPV vaccines, which after two immunizations elicit IgG maintained at stable levels 


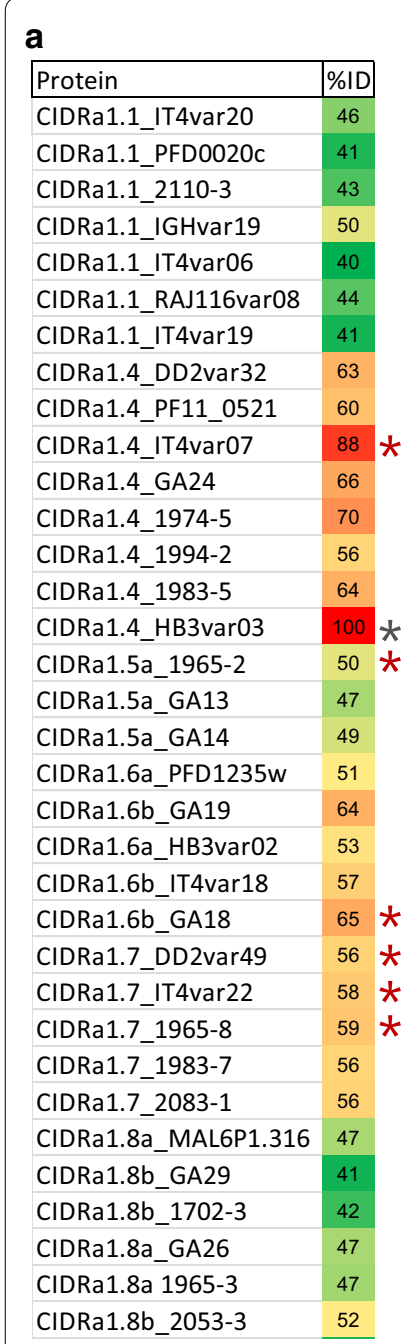

b

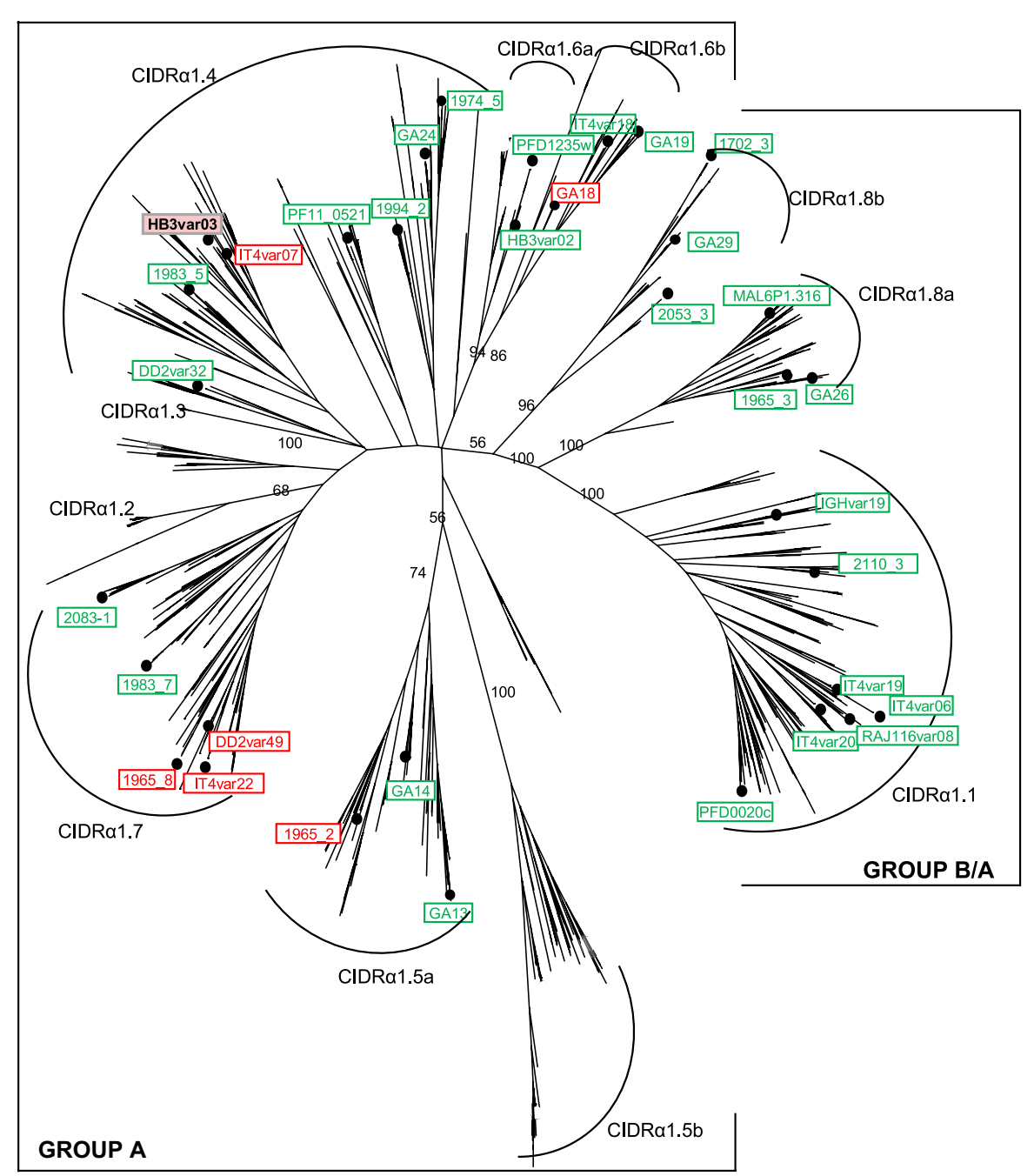

Fig. 6 Sequence analysis of CIDRa1 domains. a Pairwise sequence similarity (19 kDa sequences) of the assayed CIDRa1 domains to HB3var03 CIDRa1.4. Blue (immunogen variant) and red asterisks $\left(^{*}\right)$ mark CIDRa1 domain variants inhibited $>40 \%$. b Maximum likelihood tree (key bootstrap $[\mathrm{N}=50]$ values are indicated on branches) of $885 \mathrm{CIDRa} 1$ sequences (30 kDa) (generated in [26]). The $34 \mathrm{CIDRa} 1$ variants tested for EPCR-binding inhibition (marked with boxes) are red if inhibited $>40 \%$ by total purified lgG from animals immunized with VLP-catCIDRa1.4 or for the cognate immunogen, blue. CIDRa1 variants not inhibited are green

for decades $[29,30]$. Recently, methods that enable coupling of complex antigens to pre-formed VLP have been developed, employing the SpyTag/SpyCatcher conjugation system [31, 33, 34]. Employed in mice, these VLP vaccines have induced high and stable IgG levels against microbial antigens, including malaria antigens [48-51]. The VLP system secures a dense presentation of the vaccine antigen keeping an identical orientation on a rigid virus particle. The dense and repetitive presentation of antigens in their native conformation can override B cell tolerance and thus may alter not only the strength and longlivity of the response, but potentially also the epitopes targeted. In the case of CIDR $\alpha 1$ antigens, VLP presentain could therefore potentially alter the breadth of the immuneresponse to a broader reactivity across the protein family.

Here, the VLP-conjugated CIDR $\alpha 1.4$ vaccines induced an IgG response, which was higher and more rapid than the corresponding soluble vaccines formulated in Freund's incomplete adjuvant. The EPCR-binding inhibitory effect across diverse CIDR $\alpha 1$ domains was similar for antibodies elicited by the VLP-based and the soluble CIDR $\alpha 1.4$ vaccines. This is important as Freund's incomplete adjuvant cannot be used in humans, whereas the VLP platform is expected to be approved for human use and is likely be able to induce high tittered long-lasting immune responses in humans [52]. 
Immunizations with CIDR 1.4 fused to SpyCatcher $\mathrm{N}$-terminally appeared to induce somewhat higher levels of inhibition than CIDR $\alpha 1.4$ with a SpyCatcher fused $\mathrm{C}$-terminal. The C-terminal of CIDR $\alpha 1$ is positioned near the EPCR-binding site [26], and it is possible that a C-terminal fusion of a SpyCatcher adversely affected induction of antibodies to epitopes near or at the EPCR binding site. Overall, the VLP-presentation of CIDR $\alpha 1$ did not improve the reactivity across the CIDR $\alpha 1$ family, and it is likely that only a fraction of the elicited antibodies are functional. Additional studies testing inhibition of homologous native CIDRo1.4 PfEMP1 may further elucidate the relevance of the differences seen in EPCR binding inhibition by IgG elicited by the antigens presented on or off the VLP.

\section{Conclusions}

This study showed that immunization with VLP-conjugated CIDR $\alpha 1.4$ antigens induced a rapid and strong IgG response capable of inhibiting EPCR-binding of CIDR $\alpha 1$ proteins belonging to different CIDR $\alpha 1$ subgroups. Data from this and previous studies suggest that vaccination with a limited combination of antigens can induce an antibody response covering most EPCR-binding CIDR $\alpha 1$ variants. Future studies including in vitro studies using natively expressed PfEMP1, are needed to clarify how many variants are required to obtain broad coverage across the CIDR $\alpha 1$ protein family and if such combinations can be achieved through display of multiple antigens on vaccine platforms, such as VLP vaccines, most likely to induce potent immune responses in humans.

\section{Supplementary information}

Supplementary information accompanies this paper at https://doi. org/10.1186/s12936-020-03201-z.

Additional file 1. Raw data file. Data of IgG reactivity (MFI) and EPCAR binding (MFI) with and without presence of IgG given along with calculated level of inhition (\%).

\section{Abbreviations}

EPCR: Endothelial protein C receptor; PfEMP1: P. falciparum erythrocyte membrane protein 1; ICAM1: Intercellular adhesion molecule 1; CIDR: Cysteine-rich interdomain region; DBL: Duffy binding-like; VLP: Virus-like particle.

\section{Acknowledgements}

Susanne L. Nielsen for technical support.

\section{Authors' contributions}

$\mathrm{CH}$ analysed the data and prepared the main manuscript draft; LT and ST conducted the experimental work; AFS provided experimental tools, LT, TL and TGT conceived the experimental design and interpreted the data. All authors read and approved the final manuscript.

\section{Funding}

Financial support for this study was received from Novo Nordisk Foundation (NNF160C0023362 and NNF17OC0029344), Danish Council for Independent Research, Sapere Aude program DFF-4004-00624B and Lundbeck Foundation
(R140-2013-13448). The funders had no role in the design, analysis or interpretation of the study.

\section{Availability of data and materials}

All data generated or analysed during this study are included in this published article.

\section{Ethics approval and consent to participate}

Approval for the study was granted by the Animal Experiments Inspectorate of Denmark (Approval number: 2013-15-2934-00902/BES).

\section{Consent for publication}

Not applicable.

\section{Competing interests}

The authors have no competing interests.

\section{Author details}

${ }^{1}$ Centre for Medical Parasitology, Department of Immunology and Microbiology (ISIM), Faculty of Health and Medical Sciences, University of Copenhagen, Copenhagen, Denmark. ${ }^{2}$ Department of Infectious Diseases, Copenhagen University Hospital (Rigshospitalet), Copenhagen, Denmark.

Received: 22 October 2019 Accepted: 23 March 2020

Published online: 30 March 2020

\section{References}

1. Gupta S, Snow RW, Donnelly CA, Marsh K, Newbold C. Immunity to noncerebral severe malaria is acquired after one or two infections. Nat Med. 1999;5:340-3

2. Greenwood BM, Bradley AK, Greenwood AM, Byass P, Jammeh K, Marsh K, et al. Mortality and morbidity from malaria among children in a rural area of the Gambia, West Africa. Trans R Soc Trop Med Hyg. 1987:81:478-86.

3. Goncalves BP, Huang CY, Morrison R, Holte S, Kabyemela E, Prevots DR, et al. Parasite burden and severity of malaria in Tanzanian children. N Engl J Med. 2014;370:1799-808.

4. Bruce-Chwatt LJ. A longitudinal survey of natural malaria infection in a group of West African adults. West Afr Med J. 1963;12:199-217.

5. Marsh K, Howard RJ. Antigens induced on erythrocytes by P. falciparum: expression of diverse and conserved determinants. Science. 1986;231:150-3.

6. Bull PC, Lowe BS, Kortok M, Molyneux CS, Newbold Cl, Marsh K. Parasite antigens on the infected red cell surface are targets for naturally acquired immunity to malaria. Nat Med. 1998:4:358-60.

7. Nielsen MA, Staalsoe T, Kurtzhals JA, Goka BQ, Dodoo D, Alifrangis M, et al. Plasmodium falciparum variant surface antigen expression varies between isolates causing severe and nonsevere malaria and is modified by acquired immunity. J Immunol. 2002;168:3444-50.

8. Cham GK, Turner L, Lusingu J, Vestergaard L, Mmbando BP, Kurtis JD, et al. Sequential, ordered acquisition of antibodies to Plasmodium falciparum erythrocyte membrane protein 1 domains. J Immunol. 2009;183:3356-63.

9. Turner L, Lavstsen T, Mmbando BP, Wang CW, Magistrado PA, Vestergaard LS, et al. IgG antibodies to endothelial protein C receptor-binding cysteine-rich interdomain region domains of Plasmodium falciparum erythrocyte membrane protein 1 are acquired early in life in individuals exposed to malaria. Infect Immun. 2015;83:3096-103.

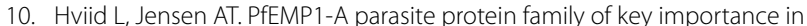
Plasmodium falciparum malaria immunity and pathogenesis. Adv Parasitol. 2015;88:51-84.

11. Marsh K, Otoo L, Hayes RJ, Carson DC, Greenwood BM. Antibodies to blood stage antigens of Plasmodium falciparum in rural Gambians and their relation to protection against infection. Trans $\mathrm{R}$ Soc Trop Med Hyg. 1989;83:293-303.

12. Giha HA, Staalsoe T, Dodoo D, Roper C, Satti GM, Arnot DE, et al. Antibodies to variable Plasmodium falciparum-infected erythrocyte surface antigens are associated with protection from novel malaria infections. Immunol Lett. 2000;71:117-26. 
13. Dodoo D, Staalsoe T, Giha H, Kurtzhals JA, Akanmori BD, Koram K, et al. Antibodies to variant antigens on the surfaces of infected erythrocytes are associated with protection from malaria in Ghanaian children. Infect Immun. 2001;69:3713-8.

14. Lusingu JP, Jensen AT, Vestergaard LS, Minja DT, Dalgaard MB, Gesase S, et al. Levels of plasma immunoglobulin $\mathrm{G}$ with specificity against the cysteine-rich interdomain regions of a semiconserved Plasmodium falciparum erythrocyte membrane protein 1, VAR4, predict protection against malarial anemia and febrile episodes. Infect Immun. 2006;74:2867-75.

15. Roberts DJ, Craig AG, Berendt AR, Pinches R, Nash G, Marsh K, et al. Rapid switching to multiple antigenic and adhesive phenotypes in malaria. Nature. 1992;357:689-92.

16. Rask TS, Hansen DA, Theander TG, Gorm Pedersen A, Lavstsen T. Plasmodium falciparum erythrocyte membrane protein 1 diversity in seven genomes-divide and conquer. PLoS Comput Biol. 2010;6:e1000933.

17. Turner L, Lavstsen T, Berger SS, Wang CW, Petersen JE, Avril M, et al. Severe malaria is associated with parasite binding to endothelial protein C receptor. Nature. 2013;498:502-5.

18. Lavstsen T, Salanti A, Jensen AT, Arnot DE, Theander TG. Sub-grouping of Plasmodium falciparum 3D7 var genes based on sequence analysis of coding and non-coding regions. Malar J. 2003;2:27.

19. Robinson BA, Welch TL, Smith JD. Widespread functional specialization of Plasmodium falciparum erythrocyte membrane protein 1 family members to bind CD36 analysed across a parasite genome. Mol Microbiol. 2003;47:1265-78

20. Jespersen JS, Wang CW, Mkumbaye SI, Minja DT, Petersen B, Turner L, et al. Plasmodium falciparum var genes expressed in children with severe malaria encode CIDRalpha1 domains. EMBO Mol Med. 2016;8:839-50.

21. Mkumbaye SI, Wang CW, Lyimo E, Jespersen JS, Manjurano A, Mosha J, et al. The severity of Plasmodium falciparum infection is associated with transcript levels of var genes encoding EPCR-binding PfEMP1. Infect Immun. 2017;85:e00841.

22. Lennartz F, Adams Y, Bengtsson A, Olsen RW, Turner L, Ndam NT, et al. Structure-guided identification of a family of dual receptor-binding PfEMP1 that is associated with cerebral malaria. Cell Host Microbe. 2017;21:403-14.

23. Magallon-Tejada A, Machevo S, Cistero P, Lavstsen T, Aide P, Rubio M, et al. Cytoadhesion to gC1qR through Plasmodium falciparum erythrocyte membrane protein 1 in severe malaria. PLoS Pathog. 2016;12:e1006011.

24. Bernabeu M, Smith JD. EPCR and malaria severity: the center of a perfect storm. Trends Parasitol. 2017;33:295-308.

25. Bouwens EA, Stavenuiter F, Mosnier LO. Mechanisms of anticoagulant and cytoprotective actions of the protein C pathway. J Thromb Haemost. 2013;11(Suppl 1):242-53.

26. Lau CK, Turner L, Jespersen JS, Lowe ED, Petersen B, Wang CW, et al. Structural conservation despite huge sequence diversity allows EPCR binding by the PfEMP1 family implicated in severe childhood malaria. Cell Host Microbe. 2015;17:118-29.

27. Fougeroux C, Turner L, Bojesen AM, Lavstsen T, Holst PJ. Modified MHC Class II-associated invariant chain induces increased antibody responses against Plasmodium falciparum antigens after adenoviral vaccination. J Immunol. 2019;202:2320-31.

28. Turner $L$, Theander $T G$, Lavstsen $T$. Immunization with recombinant Plasmodium falciparum erythrocyte membrane protein 1 CIDRa 1 domains induces domain subtype inhibitory antibodies. Infect Immun. 2018;86:e00435.

29. Kirnbauer R, Taub J, Greenstone H, Roden R, Durst M, Gissmann L, et al. Efficient self-assembly of human papillomavirus type $16 \mathrm{~L} 1$ and L1-L2 into virus-like particles. J Virol. 1993;67:6929-36.

30. Safaeian M, Porras C, Pan Y, Kreimer A, Schiller JT, Gonzalez P, et al. Durable antibody responses following one dose of the bivalent human papillomavirus $L 1$ virus-like particle vaccine in the costa rica vaccine trial. Cancer Prev Res. 2013;6:1242-50.

31. Thrane S, Janitzek CM, Matondo S, Resende M, Gustavsson T, de Jongh WA, et al. Bacterial superglue enables easy development of efficient viruslike particle based vaccines. J Nanobiotechnol. 2016;14:30.

32. Frietze KM, Peabody DS, Chackerian B. Engineering virus-like particles as vaccine platforms. Curr Opin Virol. 2016;18:44-9.

33. Brune KD, Leneghan DB, Brian IJ, Ishizuka AS, Bachmann MF, Draper SJ, et al. Plug-and-display: decoration of virus-like particles via isopeptide bonds for modular immunization. Sci Rep. 2016;6:19234.
34. Palladini A, Thrane S, Janitzek CM, Pihl J, Clemmensen SB, de Jongh WA, et al. Virus-like particle display of HER2 induces potent anti-cancer responses. Oncoimmunology. 2018;7:e1408749.

35. Cham GK, Kurtis J, Lusingu J, Theander TG, Jensen AT, Turner L. A semiautomated multiplex high-throughput assay for measuring IgG antibodies against Plasmodium falciparum erythrocyte membrane protein 1 (PfEMP1) domains in small volumes of plasma. Malar J. 2008;7:108.

36. Li L, Fierer JO, Rapoport TA, Howarth M. Structural analysis and optimization of the covalent association between SpyCatcher and a peptide tag. J Mol Biol. 2014;426:309-17.

37. Cham GK, Turner L, Kurtis JD, Mutabingwa T, Fried M, Jensen AT, et al. Hierarchical, domain type-specific acquisition of antibodies to Plasmodium falciparum erythrocyte membrane protein 1 in Tanzanian children. Infect Immun. 2010;78:4653-9.

38. Mosnier LO, Lavstsen T. The role of EPCR in the pathogenesis of severe malaria. Thromb Res. 2016;141(Suppl 2):S46-9.

39. Lavstsen T, Turner L, Saguti F, Magistrado P, Rask TS, Jespersen JS, et al. Plasmodium falciparum erythrocyte membrane protein 1 domain cassettes 8 and 13 are associated with severe malaria in children. Proc Natl Acad Sci USA. 2012;109:E1791-800.

40. Tuikue Ndam N, Moussiliou A, Lavstsen T, Kamaliddin C, Jensen ATR, Mama A, et al. Parasites causing cerebral falciparum malaria bind multiple endothelial receptors and express EPCR and ICAM-1-binding PfEMP1. J Infect Dis. 2017;215:1918-25.

41. Bertin Gl, Lavstsen T, Guillonneau F, Doritchamou J, Wang CW, Jespersen $J$ S, et al. Expression of the domain cassette 8 Plasmodium falciparum erythrocyte membrane protein 1 is associated with cerebral malaria in Benin. PLOS ONE. 2013;8:e68368.

42. Bernabeu M, Danziger SA, Avril M, Vaz M, Babar PH, Brazier AJ, et al. Severe adult malaria is associated with specific PfEMP1 adhesion types and high parasite biomass. Proc Natl Acad Sci USA. 2016;113:E3270-9.

43. Kessler A, Dankwa S, Bernabeu M, Harawa V, Danziger SA, Duffy F, et al. Linking EPCR-binding PfEMP1 to brain swelling in pediatric cerebral malaria. Cell Host Microbe. 2017;22(601-14):e5.

44. Shabani E, Hanisch B, Opoka RO, Lavstsen T, John CC. Plasmodium falciparum EPCR-binding PfEMP1 expression increases with malaria disease severity and is elevated in retinopathy negative cerebral malaria. BMC Med. 2017;15:183.

45. Petersen JE, Bouwens EA, Tamayo I, Turner L, Wang CW, Stins M, et al. Protein $C$ system defects inflicted by the malaria parasite protein PfEMP1 can be overcome by a soluble EPCR variant. Thromb Haemost. 2015:114:1038-48.

46. Rambhatla JS, Turner L, Manning L, Laman M, Davis TME, Beeson JG, et al. Acquisition of antibodies against endothelial protein $C$ receptor-binding domains of Plasmodium falciparum erythrocyte membrane protein 1 in children with severe malaria. J Infect Dis. 2019;219:808-18.

47. Hviid L, Lavstsen $T$, Jensen AT. A vaccine targeted specifically to prevent cerebral malaria-is there hope? Expert Rev Vaccines. 2018;17:565-7.

48. Bruun TUJ, Andersson AC, Draper SJ, Howarth M. Engineering a rugged nanoscaffold to enhance plug-and-display vaccination. ACS Nano. 2018;12:8855-66.

49. Leneghan DB, Miura K, Taylor IJ, Li Y, Jin J, Brune KD, et al. Nanoassembly routes stimulate conflicting antibody quantity and quality for transmission-blocking malaria vaccines. Sci Rep. 2017;7:3811.

50. Janitzek CM, Peabody J, Thrane S, Carlsen PH, Theander TG, Salanti A, et al. A proof-of-concept study for the design of a VLP-based combinatorial HPV and placental malaria vaccine. Sci Rep. 2019;9:5260.

51. Singh SK, Thrane S, Janitzek CM, Nielsen MA, Theander TG, Theisen M, et al. Improving the malaria transmission-blocking activity of a Plasmodium falciparum 48/45 based vaccine antigen by SpyTag/SpyCatcher mediated virus-like display. Vaccine. 2017;35:3726-32.

52. Romanowski B, de Borba PC, Naud PS, Roteli-Martins CM, De Carvalho NS, Teixeira JC, et al. Sustained efficacy and immunogenicity of the human papillomavirus (HPV)-16/18 AS04-adjuvanted vaccine: analysis of a randomised placebo-controlled trial up to 6.4 years. Lancet. 2009;374:1975-85.

\section{Publisher's Note}

Springer Nature remains neutral with regard to jurisdictional claims in published maps and institutional affiliations. 